\title{
Epigenetic Regulators of Mesenchymal Stem/Stromal Cell Lineage Determination
}

\author{
Dimitrios Cakouros ${ }^{1,2} \cdot$ Stan Gronthos ${ }^{1,2}(D$
}

Published online: 13 August 2020

(C) The Author(s) 2020

\begin{abstract}
Purpose of Review Although many signalling pathways have been discovered to be essential in mesenchymal stem/stromal (MSC) differentiation, it has become increasingly clear in recent years that epigenetic regulation of gene transcription is a vital component of lineage determination, encompassing diet, lifestyle and parental influences on bone, fat and cartilage development. Recent Findings This review discusses how specific enzymes that modify histone methylation and acetylation or DNA methylation orchestrate the differentiation programs in lineage determination of MSC and the epigenetic changes that facilitate development of bone related diseases such as osteoporosis. The review also describes how environmental factors such as mechanical loading influence the epigenetic signatures of MSC, and how the use of chemical agents or small peptides can regulate epigenetic drift in MSC populations during ageing and disease.

Summary Epigenetic regulation of MSC lineage commitment is controlled through changes in enzyme activity, which modifies DNA and histone residues leading to alterations in chromatin structure. The co-ordinated epigenetic regulation of transcriptional activation and repression act to mediate skeletal tissue homeostasis, where deregulation of this process can lead to bone loss during ageing or osteoporosis.
\end{abstract}

Keywords Mesenchymal stem/stromal cells $\cdot \mathrm{MSC} \cdot$ Epigenetics $\cdot$ Skeletal stem cells $\cdot$ DNA methylation $\cdot$ Histone methylation $\cdot$ Histone acetylation

\section{Introduction}

The stromal component within bone marrow is a hierarchical continuum of mature functional stromal populations and committed progenitor cells. This is sustained by a minor population of long-lived, self-renewing and multipotential skeletal stem cells or bone marrow-derived mesenchymal stem/ stromal cells (BMSC) [1]. Purified preparation of human BMSC exhibit the potential to form osteoblasts, adipocytes, chondrocytes, smooth muscle cells and myelosupportive

This article is part of the Topical Collection on Skeletal Biology and Regulation

Stan Gronthos

stan.gronthos@adelaide.edu.au

1 Mesenchymal Stem Cell Laboratory, Adelaide Medical School, Faculty of Health and Medical Sciences, University of Adelaide, Adelaide, SA, Australia

2 Precision Medicine Theme, South Australian Health and Medical Research Institute, Adelaide, SA, Australia fibroblasts under inductive conditions in vitro or when transplanted in vivo [2-4]. It is well known that the master regulatory transcription factors Runt-related transcription factor 2 (RUNX2), peroxisome proliferator-activated receptor gamma 2 (PPAR $\gamma 2$ ), myogenic differentiation (MOYD) and sex-determining region Y-box 9 (SOX9) are critical mediators of BMSC differentiation towards the osteogenic, adipogenic, myogenic and chondrogenic lineages, respectively [5-7]. The co-ordinated expression of RUNX2, PPAR $\gamma 2$, MYOD or SOX9 depends on the regulation of chromatin, allowing the activation of these genes and their targets. Therefore, it is critically important to understand the epigenetic modifications regulating BMSC cell fate and lineage-specification.

Epigenetics is the cellular modification of reversible and heritable changes in gene expression that occur without changes in the DNA code [8]. Epigenetic modifications such as DNA methylation and histone modifications regulate the structure of chromatin, which determines the accessibility of genes to transcription factors and other modulators involved in gene regulation. Chromatin is formed through the compaction of DNA strands wrapped around nucleosomes [9], which are 
linked by histone proteins. The functional state of chromatin, whether open or compact, can be modified through methylation of DNA at cytosines within $\mathrm{CpG}$ dinucleotides and on amino acid residues within the histone proteins. DNA methylation is often associated with gene silencing, whereas histone modifications can be mediators of either gene activation or repression. This review will summarise our current understanding of the association and role of epigenetic modifications in BMSC lineage commitment.

\section{Enzymes Regulating Histone Methylation/Acetylation in BMSC Differentiation}

The histone 3 lysine 27 (H3K27) methyltransferase, EZH2, is essential for embryogenesis and postnatal tissue development [10-12]. We have recently reported an essential function of EZH2 as an epigenetic switch mediating the function of human BMSC. In human BMSC, EZH2 is a promoter of adipogenesis and inhibitor of osteogenesis via tri-methylation of H3K27 (H3K27me3) of Runx2 and osteopontin genes [13••]. In contrast, the H3K27me3 demethylase, KDM6A, was found to inhibit BMSC adipogenesis and promote osteo/chondrogenesis [13••]. Similarly, another H3K27me3 demethylase, KDM6B, was also discovered to be essential in osteogenic differentiation of MSC [14-17]. We and others have recently expanded these studies in conditional knockout mice where EZH2 is specifically deleted in BMSC leading to increased bone formation with disrupted bone patterning, increased trabecular bone, and increase bone marrow fat $[18$, 19]. The role of EZH2 in murine adipogenesis acts by binding to $\beta$-catenin associated genes, Wnt1, 6, 10a, 10b, 2b, 3a, 8a, 2 and 11 in peripheral pre-adipocytes and their repression promotes adipogenesis [20]. In a mouse model of osteoporosis induced by oestrogen deficiency following bilateral ovariectomy, EZH2 protein and RNA levels were found to be elevated in BMSC isolated from osteoporotic bone samples, with EZH2 and H3K27me3 being highly enriched on promoters of Wnt1, Wnt6 and Wnt10a leading to repression of these bone promoting genes [21] and promoting adipogenesis. Therefore, EZH2/KDM6A/B represent a novel epigenetic switch for controlling BMSC cell fate decisions, part of a complex circuitry network of epigenetic regulators (Fig. 1).

Methylation of histone 3 on lysine 4 ( $\mathrm{H} 3 \mathrm{~K} 4 \mathrm{me} 3)$ is associated with transcriptional activation and pivotal for differentiation [22, 23]. Absent, small or homeotic disc1 like (Ash11) is a member of the Trx family that methylates H3K4. Both Ash1l and H3K4me3 were upregulated in multipotent mouse C3H10T1/2 cells or human BMSC cells undergoing osteogenic, adipogenic or chondrogenic differentiation [24]. Depletion of Ash11 impaired osteo/chondrogenic differentiation but increased adipogenesis, due to a decrease in Hoxa10,
Sox9 gene expression and an activation of PPAR $\gamma 2$ expression [24]. Of note, Ash11 and H3K4me3 were found to be downregulated in murine and human osteoporotic bone samples. Ash 11 cooperates with other H3K4 methyltransferases such as MLL1 [25, 26], which activate Hox genes [27] involved in osteogenesis. The H3K4 demethylase, RBP2, occupies the osteoblast-specific transcription factor Osterix transcription start site to remove H3K4me3 [28]. Conversely, the H3K 4 methyltransferase Set1, part of the COMPASS complex (consisting of MLL, KDM6A, Set1, WDR5), deposits H3K4me3 on the Osterix promoter during osteogenesis [29]. Similarly, the co-factor WDR5 can induce H3K4me3 on the Runx2 promoter, whereas KDM6A removes H3K27me3 during osteogenesis [30]. The balance between competing epigenetic factors is further illustrated by the demethylase, KDM5A, which removes $\mathrm{H} 3 \mathrm{~K} 4 \mathrm{me} 3$ and inhibits osteogenesis by repressing Runx2 expression [31]. In contrast, KDM7C was shown to promote osteogenesis by enhancing Runx2 binding to osteogenic promoters via demethylation of the Runx2 gene [32].

Another important epigenetic modification is methylation of histone 3 lysine 9 (H3K9me), a marker for gene repression. The H3K9me demethylase, KDM4A, was found to promote adipogenesis and conversely inhibit osteogenesis in primary cultures of BMSC [33]. Subsequent investigations revealed that the Wnt pathway was directly inhibited by KDM4A. However, KDM4A can also act indirectly on bone by reducing DNA methylation on the promoters of the adipogenic associated factors, $\mathrm{C} / \mathrm{EBP} \alpha$ and SFRP4, leading to activation of adipocyte differentiation. In other investigations, knockout of the H3K9 methyltransferase, SETDB1, was found to cause bone defects in mice [34], implying that SETDB1 is a promoter of osteogenesis. In contrast, knockdown of SETDB1 expression resulted in increased adipogenesis in vitro [35], indicative of another epigenetic mediator of BMSC lineage commitment via $\mathrm{H} 3 \mathrm{~K} 9$ modification of osteogenic and adipogenic associated gene sets.

Some epigenetic enzymes exhibit different functional roles targeting more than one epigenetic mark such as NO66, a H3K4 and H3K36 demethylase, which has been shown to bind to the Osterix promoter and inhibit transcription [36]. NO66 conditional knockout mice, in the MSC lineage, exhibit increased length and body weight, which is associated increased intramembranous and endochondral ossification [37]. Similarly, the demethylase, KDM7A, was reported to promote adipogenesis but inhibit osteogenesis [38] through demethylation of both H3K9me2 and H3K27me2. KDM7A action resulted in an activation of gene expression levels for the adipogenic associated factors, C/EBP $\alpha$ and SFRP1 a known Wnt inhibitor.

Histone acetylation is an epigenetic modification associated with transcriptional activation, where histone deacetylation causes chromatin to compact, leading to transcriptional 
Fig. 1 Epigenetic switches regulating skeletal stem cell fate determination. Known enzymes mediating skeletal stem cell osteogenic, chondrogenic or adipogenic commitment. (?) Conflicting functions
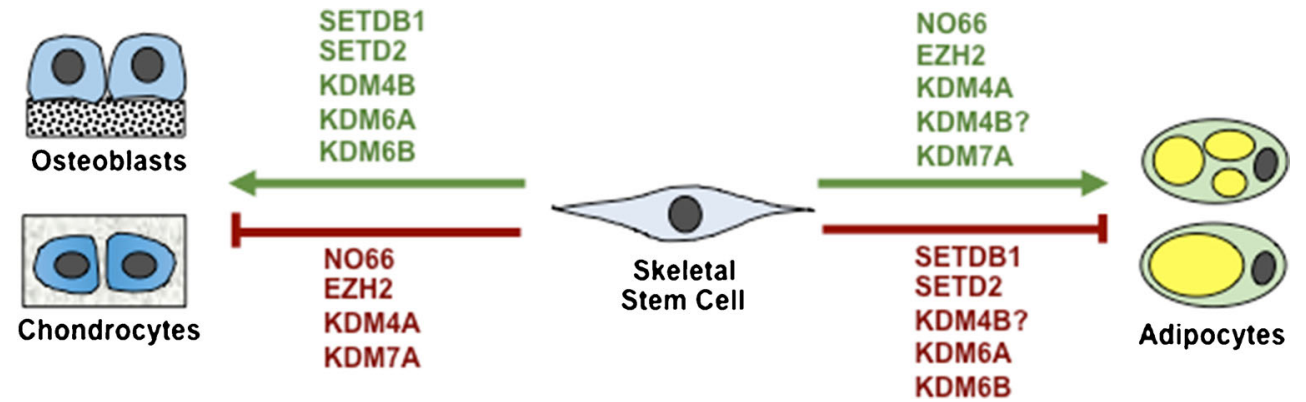

repression [39, 40]. Members of the histone deacetylases (HDAC), HDAC 3, 4, 5 and 7 are critical for endochondral bone formation [41, 42]. HDAC3 deletion in chondrocytes leads to embryonic lethality, whereas postnatal deletion of HDAC3 delays ossification, growth plate maturation with increased osteoclast activity [43]. The phenotype was associated with increased cytokine and matrix-degrading genes due to increased NF- $\mathrm{KB}$ acetylation and decreased bone development genes [43]. An epigenetic library screen identified Abexinostat, which increased H3K9Ac levels on osteogenic/ adipogenic genes and promoted both osteogenic and adipogenic differentiation [44••]. This study highlights the utility of screening chemical and small peptide libraries in order to develop new-generation agents to modify histone acetylation patterns controlling MSC cell fate determination (Fig. 2).

Investigations into mechanical bone stimulation demonstrated enhanced Notch signalling and downregulated HDAC1, in human osteoporotic BMSC [45••]. HDAC1 was found to inhibit bone formation and Notch signalling via deacetylating JAG1 and inhibiting HDAC1 attenuated hind limb unloading induced osteoporosis [45••]. Aged BMSC are characterised by reduced differentiation and proliferation, processes which are known to be mediated by HDAC and longevity genes, such as the sirtuins. The mitochondrial deacetylase, Sirt3, was found to be downregulated during culture expansion but was a promoter of both osteogenic and adipogenic differentiation, and attenuated oxidative stress and senescence [46]. Knockout studies of another deacetylase, Sirt7, found that mice developed osteopenia as Sirt7 interacted with Osterix, deacetylating K368, increasing its transactivation and function [47]. A recent study reported that
HDAC4 deficient mice display premature ossification due to early-onset chondrocyte hypertrophy [48]. Similarly, HDAC3 deletion specifically in chondrocytes caused delayed angiogenesis, increased bone resorption and severely reduced bone median density [43]. HDAC3 was found to be essential for Collagen type 2 gene expression, while repressing NF- $\mathrm{KB}$ and STAT signalling [43].

To date, the gene promoter of the osteogenic master regulatory factor, Runx2, has been reported to undergo specific changes in epigenetic modifications including, enrichment of histone 3 acetylation ( $\mathrm{H} 3 \mathrm{Ac}), \mathrm{H} 3 \mathrm{~K} 4 \mathrm{me} 3$ and reduced H3K4me1, H3K27me3 and H4R3me2 [49]. Chromatin immunoprecipitation (ChIP) analysis showed increased binding of WDR5 and KDM6A on the Runx2 promoter during osteogenesis. The enzymes MLL2, MLL3 and Menin, part of the MLL2/MLL3/COMPASS complex, were also found to be present and acted to promote $\mathrm{H} 3 \mathrm{~K} 4 \mathrm{me} 3$ to activate Runx2 transcription. Amongst the H3K4 demethylases, LSD1, a flavin-containing amino oxidase that removes $\mathrm{H} 3 \mathrm{~K} 4 \mathrm{me} 1$ and $\mathrm{K} 4 \mathrm{me} 2$ was shown to be an inhibitor of osteogenesis in human adipose-derived stem cells. Furthermore, conditional deletion of LSD1 in MSC resulted in a phenotype of short stature, lower body weight, delayed cartilage development and endochondral bone formation, with bones showing increased trabecular and cortical bone volume with increased osteoblast activity. ChIPseq analysis of calvarial cells showed increased H3K4me2 levels with Gene Ontogeny pathway analysis indicating many genes as being involved in bone differentiation including Wnt7b and Bmp2 [50••]. The H3K36me3 mark is a modification orchestrated by SETD2, which is positively correlated with transcription, prevalent on gene bodies and associated with longevity. SETD2 and
Fig. 2 Histone deacetylases regulating skeletal stem cell fate determination. Known histone deacetylase enzymes regulating osteogenesis, adipogenesis and chondrogenesis
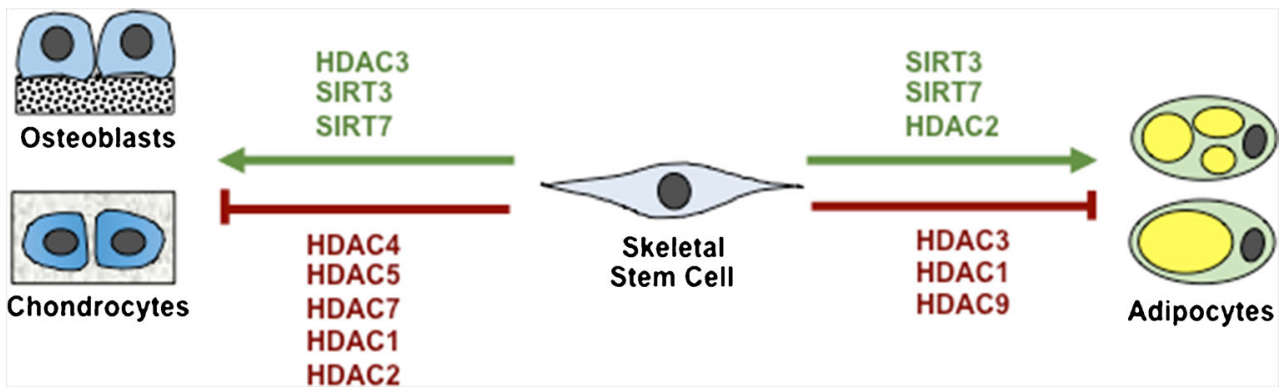

HDAC2 
H3K36me3 levels have been shown to decrease during adipogenesis but increase during osteogenesis [51]. MSC specific conditional knockouts found that SETD2 inhibits adipogenesis and PPAR $\gamma 1 / 2, \mathrm{C} / \mathrm{EBP} \alpha$ and Fabp4 gene expression and promotes osteogenesis. Moreover, SETD2 knockout mice showed increased marrow fat and reduced bone mass (both trabecular and cortical) in distal femurs indicative of ageassociated osteoporosis.

Collectively, these studies demonstrate that the interplay between different epigenetic factors targeting histones is delicately balanced in order to orchestrate correct developmental pathways by targeting lineage-specific gene sets that mediate BMSC cell fate determination.

\section{Enzymes Regulating DNA Methylation/Hydroxylation in BMSC Differentiation}

There is overwhelming evidence implicating DNA methylation as an inhibitor of BMSC osteogenic and adipogenic differentiation in vitro [52・, 53, 54]. DNA methylation has also been shown to affect mechanical bone loading, acting to maintain bone density in vivo, with unloading leading to increased DNA methylation, bone loss and disuse osteoporosis $[55,56]$. Biomechanical force has been found to induce important BMSC lineage pathways such as Wnt and Bmp signalling $[57,58]$ and more recently the Sonic hedgehog pathway (SHH) [59]. The DNA methyltransferase, DNMT3B, is reported to be upregulated at the initial stage of fracture repair and mainly expressed in chondrogenic progenitors, where chondroblast specific deletion of DNMT3B results in diminished fracture repair. Whilst the promoter of SHH was found to be hypermethylated, methylation levels decreased following mechanical stimulation. Moreover, DNMT3B was discovered to methylate the SHH gene promoter, and was dissociated from the promoter following mechanical stimulation, leading to a reduction of methylation levels and gene activation [59].

In more recent years, the hydroxylases, Ten-eleventranslocases (Tet1, Tet2 and Tet3) have emerged as key regulators of stem cell renewal. They catalyse the conversion of 5methylcytosine $(5 \mathrm{mC})$ into 5 -hydroxymethylcytosine $(5 \mathrm{hmC})$ where it opposes the function of $5 \mathrm{mC}$ and leads to DNA demethylation [60-62]. In human BMSC, Tet1 was found to be repressed during osteogenesis and adipogenesis, whereas Tet2 was found to promote both osteogenesis and adipogenesis [52••]. It should be noted that both Tet1 and Tet2 were found to be downregulated in human and murine BMSC derived from osteoporotic bone samples, correlating to reduced $5 \mathrm{hmC}$ levels in situ $[52 \bullet \bullet, 63 \bullet \bullet$. Mechanistically, Tet2 was found to hydroxymethylate Runx2 and Bmp2, resulting in activation of transcription. In other studies, however, both
Tet 1 and Tet 2 were found to promote both osteogenesis and chondrogenesis in murine mesenchymal cells [64] and murine BMSC. This occurred via demethylation of $\mathrm{P} 2 \mathrm{rX} 7$, which was found to act as an activator of Runx2 gene expression [63・•]. In vivo analyses showed deletion of both Tet1 and Tet2 resulted in impaired self-renewal of murine BMSC, and was associated with a severe osteopenic phenotype. Studies of chondrogenesis revealed that global $5 \mathrm{hmC}$ levels increased over time correlating to increased expression levels of Tet1, Tet2 and Tet3 [65]. Knockdown of Tet1 had the greatest effect on $5 \mathrm{hmC}$ levels. Highest levels of $5 \mathrm{hmC}$ were observed in gene bodies and in promoters preceding transcription start sites on chondrogenic genes such as Sox5, Sox6 and Sox9. Metaanalysis of genes acquiring $5 \mathrm{hmC}$ identified pathways essential to cartilage development such as the WNT pathway and combining expression data with $5 \mathrm{hmC}$ data showed a close correlation between $5 \mathrm{hmC}$ and transcription.

The complexity of BMSC cell fate determination is dependent on interacting epigenetic modifications that control key lineage-specific transcription factors. Osterix is one of the most important lineage determinants for bone formation and is expressed specifically by pre-osteoblasts and chondrocytes [1, 66]. Examination of epigenetic modifications on the Osterix promoter showed that MSC differentiation into osteoblasts, correlated to enriched levels of $\mathrm{H} 3 \mathrm{Ac} / \mathrm{H} 3 \mathrm{~K} 4 \mathrm{me} 3$ accompanied by a decrease in H3K9me3/H3K27me3 [29]. In non-mesenchymal cells, the Osterix promoter was found to be enriched in SUV39H1 and EZH2, which deposit H3K9me3 and H3K27me3 modifications, respectively. Moreover, the analysis of MSC identified several epigenetic modifiers, RNAPII, HDAC1/2/4, Setdb1, JMJD2a, EZH2, JMJD3 and KDA6A bound on the Osterix promoter [29]. Osteogenic differentiation caused enrichment of RNAPII, JMJD2a and JMJD3 and reduced HDAC1/2/4, Setdb1 and EZH2 and DNA methylation due to the activity of DNMT1 and DNMT3A. Higher $5 \mathrm{hmC}$ levels were also detected on the Osterix promoter when $5 \mathrm{mC}$ was reduced. Overall, there was an observed reduction in histone $\mathrm{H} 3$ levels indicative of chromatin remodelling during osteogenesis. Chromatin remodelers, Brg1 and Brm, were found at high levels on the Osterix promoter in MSC and osteoblasts but not in non-MSC cells, suggesting chromatin remodelling allows transcription to occur in permissive cells. Of note, both Tet1 and Tet2 levels were also enhanced during osteogenesis and were associated with increased binding to the Osterix promoter. Moreover, knockdown of Tet 1 and Tet 2 revealed elevated $\mathrm{H} 3$ on the promoter, while Brg1, Brm, Jmjd2a, H3Ac and H3K4me3 levels were reduced, and repressive marks such as H3K9me3 and H3K27me3 were enhanced. The binding of COMPASS complex, WDR5 and SETD1, MLL2 and MLL4, was also dependent on Tet1 and 2 indicating that 
the Tet molecules act as scaffolding proteins recruiting epigenetic modulators to the promoter sites to facilitate BMSC lineage determination [29].

\section{Genome-Wide Epigenetic Changes During MSC Lineage Determination}

Identifying genome-wide epigenetic signature changes and chromatin remodelling regions during BMSC multidifferentiation enables the identification of essential genes, promoters and enhancers that function in lineage determination. Studies of histone modifications have been examined using C3H10T1/2 cells and BMSC following osteogenic differentiation in vitro [67]. Interestingly, these studies reported that global histone modification levels remained largely unchanged. Examination of osteogenic genes by ChIP analysis upstream of the transcription start site, promoter and exon regions found Runx2 to exhibit no observable changes in occupancy of histones. However, Osterix, alkaline phosphatase, bone sialoprotein and osteocalcin all showed decreases in histone 3 upstream of and including the promoters [67]. Furthermore, H3K9 and H3K14 acetylation increased upstream of the transcription start site and promoter region for all genes. Whilst no differences were seen in $\mathrm{H} 3 \mathrm{~K} 4 \mathrm{me} 1$ and H3K4me3 levels, H3K4me2 increased upstream of transcription start site for Runx2 and Osterix. In contrast, H3K9me2 and H3K27me3 levels decreased for both Runx2 and Osterix. When examining BMSC derived from osteoporotic samples, there were low levels of $\mathrm{H} 3 \mathrm{~K} 9$ and $\mathrm{H} 3 \mathrm{~K} 14$ acetylation and H3K4me2, and higher levels of H3K27me3 on Runx2 and Osterix promoters and upstream regions correlating with reduced transcription.

Whole-genome histone modifications and DNA methylation have been mapped during chondrogenic differentiation of human BMSC [68]. The transcriptional permissive marks H3K4me3, H3K9Ac and H3K36me3 showed similarity between donors. While H3K4me3 and H3K9Ac levels were upregulated in activated genes, H3K36me3 showed the strongest correlation, whereas $\mathrm{H} 3 \mathrm{~K} 27 \mathrm{me} 3$ was increased in repressed genes. H3K27me3 appeared to decrease in chondrogenic associated genes. Downregulated genes showed a decrease in H3K4me3, H3K9Ac and H3K36me3, with the majority of activated genes showing increased H3K4me3 levels. Gene ontology analysis of H3K4me3 genes showed enrichment for skeletal development, extracellular matrix molecules and chondrocyte differentiation. Genes with downregulated H3K4me3 were enriched for apoptosis, development and metabolism pathways. Genes that were not upregulated but had H3K4me3 did not exhibit increased levels of $\mathrm{H} 3 \mathrm{~K} 9 \mathrm{Ac}$ or H3K36me3. Based on the enhancer marks $\mathrm{H} 3 \mathrm{~K} 4 \mathrm{me} 1$ and H3K27Ac, the majority of upregulated genes were in the vicinity of enhancers. Chondrogenic signature genes were found to be hypomethylated before and after differentiation illustrating that DNA methylation has no significant role in chondrogenesis. A similar study examining chondrogenesis in vitro employed a gene stratification analysis into low, middle and high expression. Histone methylation marks H3K4me3, H3K27Ac, H3K4me1 and H3K36me3 were associated with high expressing genes [69]. Conversely, $\mathrm{H} 3 \mathrm{~K} 27 \mathrm{me} 3$ was associated with low expressing genes. In differentiated chondrocytes, the H3K4me1 and H3K27Ac enhancer marks correlated with chondrogenesis and cartilage function. When compared to the NIH road map, which entails chromatin states of over a hundred different cell types, there was no significant clustering when excluding enhancer marks. When enhancer marks were examined, they clustered with similar cell types such as chondrocytes derived from BMSC. In addition, the Human Methylation 450k bead ChIP array was used to examine DNA methylation showing demethylation predominantly on genes related to chondrogenesis. This is in contrast to other studies that showed limited DNA methylation changes, which were biased towards promoters.

Genome-wide epigenetic map analysis during adipogenesis, using human adipose-derived MSC and embryonic derived 3T3L1 cells, found increased $\mathrm{H} 3 \mathrm{~K} 4 \mathrm{me} 3$ levels along with gene promoters during adipogenesis, while $\mathrm{H} 3 \mathrm{~K} 4 \mathrm{me} 1$ and H3K27Ac marks were found mainly in promoters, introns and intergenic regions [70]. H3K36me3 levels were found across transcribed bodies that increased during adipogenesis. The repressive mark, H3K27me3, was distributed broadly associated with inactive genes. Moreover, H3K4me1 and H3K27Ac levels changed dramatically during differentiation in adipogenic regulated genes, correlating to increased adipocytes, and H3K27Ac levels in all regions. In a study examining the adipogenic/osteogenic potential of murine BMSC, the ability of the cells to transdifferentiate from adipocytes to osteoblasts was evident at the epigenetic level, implying a plasticity property following lineage maturation [71].

Gene ontology analyses investigating changes in epigenetic profiles of murine BMSC during osteogenic differentiation revealed genes associated with cell cycle, DNA replication and non-bone lineage transcription factors were downregulated, whereas bone differentiation genes were upregulated [72]. Highly upregulated genes showed some changes in $\mathrm{H} 3 \mathrm{~K} 4 \mathrm{me} 3$ but more so with H3K9Ac. The H3K27Ac and H3K36me3 marks showed a closer relationship, which increased for upregulated genes and decreased for downregulated genes. In addition, most upregulated genes showed a decrease in H3K27me3 levels, whereas downregulated genes exhibited no overall changes in H3K27me3 levels. Genome-wide DNA methylation profiles during osteogenic differentiation of human BMSC indicate that the UTR region of genes showed no change in methylation whereas the promoter, exon, intron and intergenic regions showed significant changes [73]. Binding sites for transcription factors involved in maintaining 
the immature phenotype were hypermethylated, in contrast to differentiation inducing transcription factors, which were largely hypomethylated. Therefore, initiation of MSC commitment requires a co-ordinated suppression of genes associated with MSC stemness, whilst at the same time allowing the opening of chromatin containing differentiation-associated genes for transcription to occur. This process appears to be dependent on epigenetic modification patterns at specific sites along with whole genes.

\section{Therapeutic Targeting of Epigenetic Regulators in Osteoporosis}

The use of EZH2 inhibitor GSKJ126 reduces H3K27me3 around transcription start sites, enhancing expression of osteogenic initiating factors, PTH, BMPs, Wnt genes and extracellular matrix genes in murine MC3T3 cells [74••], resulting in enhanced mineral formation. Micro-computed tomography and histomorphological analyses showed that GSKJ126 treatment of mice increased cortical bone thickness in 2-month-old femora as well as osteoblast numbers. This was also seen in an ovariectomy mouse model as GSKJ126 mitigated bone loss, increased cortical and trabecular bone in femurs of ovariectomised mice. Furthermore, studies of BMSC isolated from bone samples of osteoporotic mice following bilateral ovariectomy demonstrated that $\mathrm{EZH} 2$ was overexpressed in BMSC resulting in increased levels of the repressive mark H3K27me3 on Wnt1, 6 and 10a [21]. Treating osteoporotic bone-derived MSC with the EZH2 chemical inhibitor, DZnep, increased Wnt expression and enhanced osteogenesis whilst reducing adipogenesis [21].

An osteoporotic model based on injecting dexamethasone into the tibia of mice showed an increase in adipogenesis and expression of adipogenic genes, PPAR $\gamma 2, \mathrm{C} / \mathrm{EBP} \alpha$ and GLUT4, and a reduction in osteogenic genes Runx2, alkaline phosphatase, bone sialoprotein and Osteocalcin [75]. Increased DNA demethylation was observed on the PPAR $\gamma 2$ promoter along with increases in $\mathrm{H} 3 \mathrm{~K} 9, \mathrm{H} 3 \mathrm{~K} 14$ and $\mathrm{H} 3 \mathrm{~K} 12$ acetylation in MSC derived from osteoporotic bone samples. H3K9me2 levels also decreased corresponding to a decrease in HDAC1, SETDB1 and an increase in the H3K9 demethylase, LSD1. Other studies examining induced bone loss in the femora of rats, due to the lack of mechanical loading, reported a decrease in the long coding RNA H19 coinciding with increased $\mathrm{CpG}$ methylation along with its promoter [76] due to an increase in DNMT1 activity. H19 activates the Wnt and ERK pathways hence its decrease leads to decreased osteogenesis. Studies of siRNA mediated knockdown of DNMT1 gene expression in the femora of rats were also found to enhance osteogenesis and trabecular number and bone volume.

The H3K36me3 demethylase, SETD2, has been shown to be downregulated during adipogenesis, whereas SETD2 levels increase during osteogenesis [51]. Analysis of SETD2 deficiency in the mesenchymal lineage showed enhanced adipogenesis and reduced osteogenesis, where SETD2 knockout mice exhibited reduced cortical bone, trabecular bone number and bone volume, akin to an osteoporotic-like phenotype. Purified BMSC from these mice showed differential changes in gene expression patterns, correlating to a dramatic reduction in $\mathrm{H} 3 \mathrm{~K} 36 \mathrm{me} 3$ levels mainly occurring in gene bodies, promoters, $3^{\prime} \mathrm{UTR}$, and intergenic regions [51]. Furthermore, SETD2 was found to be decreased in aged murine BMSC (20-60 weeks), supporting other findings that $\mathrm{H} 3 \mathrm{~K} 36 \mathrm{me} 3$ promotes longevity [51].

The H3K4 methylation mark is associated with gene activation, where the H3K4 demethylase, KDM5A, was found to be specifically upregulated in both human and mouse BMSC derived from osteoporotic bone samples [31]. Functional studies demonstrated that overexpression of KDM5A causes inhibition of BMP-2 induced BMSC osteogenesis. ChIP analysis of chromatin isolated from KDM5A overexpressing BMSC found lower levels of $\mathrm{H} 3 \mathrm{~K} 4 \mathrm{me} 3$ on the Runx2 promoter. The KDM5A mediated inhibition of BMSC osteogenic differentiation was

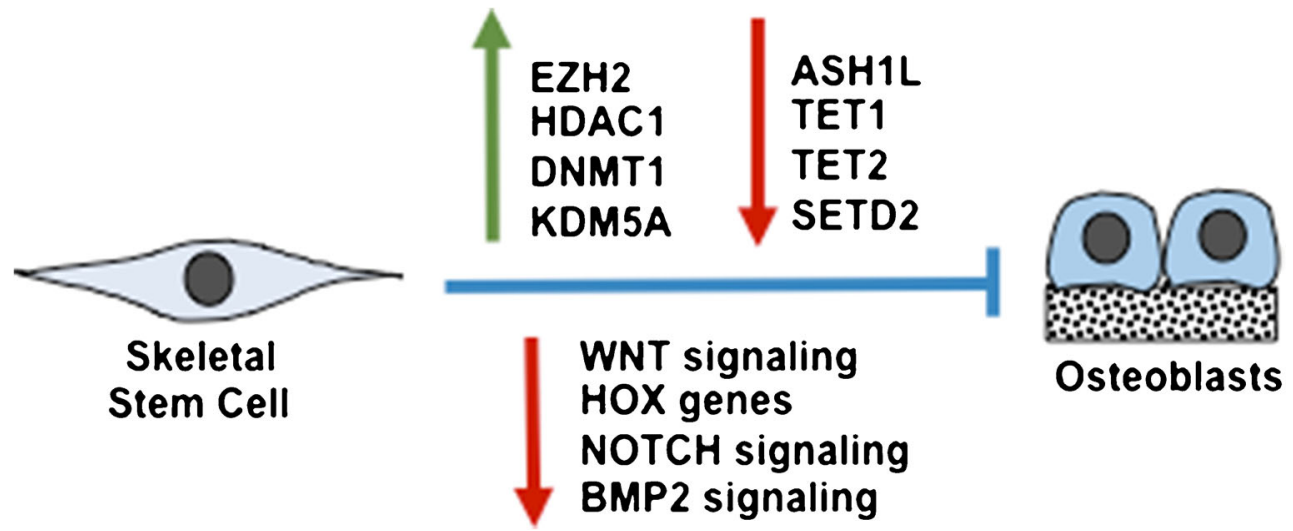

Fig. 3 Epigenetic enzymes deregulated during osteoporosis. Epigenetic enzymes deregulated in skeletal stem cells during the onset of osteoporosis leading to reduced osteoblast numbers and function. Upregulation of EZH2 and upregulation of DNMT1 inhibits WNT signalling; downregulation of ASH1L 1 suppresses HOX genes; upregulation of HDAC1 inhibits Notch signalling; downregulation of TET1/2 suppresses Runx2; downregulation of SETD2 and upregulation of KDM5A inhibits BMP2 signalling 
restored by the addition of specific KDM5A short hairpin RNA or inhibitor. Moreover, bone loss in osteoporotic mice, was partly rescued following pre-treatment with a chemical inhibitor to KDM5A activity, demonstrating the regulatory role of KDM5A in osteoporosis in mice [31].

Collectively, these preclinical studies highlight the reversible nature of the epigenetic landscape under pathological settings, which is amenable to drug targeting (Fig. 3). However, it remains to be determined whether chemical inhibitors targeting epigenetic factors are indeed specific to the appropriate enzymes, where many of these are currently being assessed in human cancer trials. The issue of targeting skeletal tissue using bone-specific carrier vehicles is also currently under investigation using various approaches. Finally, the activity of epigenetic inhibitors to prevent bone loss in diseases such as osteoporosis must be considered with caution due to unexpected consequences such as the potential to diminish the function or pool of skeletal stem cells over time.

\section{Conclusions}

Epigenetic regulation of MSC differentiation is an area that has progressed rapidly in the last decade with the use of technologies such as ATAC-seq, ChIP seq, bisulphite sequencing and 3C-chromatin capture. With more than 20 known histone modifications and two key DNA modifications, the potential for unique epigenetic combinations is staggering, illustrating the field is still in its infancy. The literature up to now shows that during differentiation, activating histone modifications consisting of combinations of H3K4me1/2/3, H3K36me3, H3R17, H3K9Ac and H3K27Ac prevail along with lineagespecific genes related to osteogenesis, adipogenesis and chondrogenesis, depending on the differentiation path. The most tightly associated mark with lineage transcription is H3K36me3/H3K4me3 along with gene bodies and transcription start sites together with $\mathrm{H} 3 \mathrm{~K} 4 \mathrm{me} 1$ and H3K27Ac that mark enhancers. The enhancer modifications closely correlate with active lineage-associated transcription and are sites of active chromatin remodelling. Commonly seen is the appearance of repressive marks (H3K27me2/3, H3K9me2) on lineages not activated to ensure their repression. Although DNA methylation on promoters is not entirely consistent with BMSC differentiation, DNA methylation along with gene bodies and enhancers is closely linked with transcription as removal of DNA methylation marks is associated with activation. Epigenetic deregulation in osteoporotic BMSC implies loss of cell identity with representation of non-bone markers, compensatory mechanisms cause an increase in early osteogenic gene expression to synthesise more bone and changes in the active and repressive histone marks and DNA methylation on lineage-associated genes lead to decreased osteogenesis and enhanced adipogenesis. Further investigations into the identification of novel epigenetic factors and target genes regulating MSC differentiation will help progress the development of epigenetic drug-specific targeting of skeletal tissues for a range of orthopaedic-related indications, by coupling with new drug screening and drug delivery approaches.

Funding Information This review was supported in part by the National Health and Medical Research Council, Australian Federal Government Project Grant APP1141361.

\section{Compliance with Ethical Standards}

Conflict of Interest The authors declare that they have no conflict of interest.

Open Access This article is licensed under a Creative Commons Attribution 4.0 International License, which permits use, sharing, adaptation, distribution and reproduction in any medium or format, as long as you give appropriate credit to the original author(s) and the source, provide a link to the Creative Commons licence, and indicate if changes were made. The images or other third party material in this article are included in the article's Creative Commons licence, unless indicated otherwise in a credit line to the material. If material is not included in the article's Creative Commons licence and your intended use is not permitted by statutory regulation or exceeds the permitted use, you will need to obtain permission directly from the copyright holder. To view a copy of this licence, visit http://creativecommons.org/licenses/by/4.0/.

\section{References}

Papers of particular interest, published recently, have been highlighted as:

•- Of major importance

1. Owen M, Friedenstein AJ. Stromal stem cells: marrow-derived osteogenic precursors. CIBA Found Symp. 1988;136:42-60.

2. Gronthos S, Zannettino AC, Hay SJ, Shi S, Graves SE, Kortesidis A, et al. Molecular and cellular characterisation of highly purified stromal stem cells derived from human bone marrow. J Cell Sci. 2003;116(Pt 9):1827-35.

3. Shi S, Gronthos S. Perivascular niche of postnatal mesenchymal stem cells in human bone marrow and dental pulp. J Bone Miner Res. 2003;18(4):696-704.

4. Zannettino AC, Paton S, Kortesidis A, Khor F, Itescu S, Gronthos S. Human mulipotential mesenchymal/stromal stem cells are derived from a discrete subpopulation of STRO-1bright/CD34 /CD45(-)/glycophorin-A-bone marrow cells. Haematologica. 2007;92(12):1707-8.

5. Ducy P, Zhang R, Geoffroy V, Ridall AL, Karsenty G. Osf2/Cbfa1: a transcriptional activator of osteoblast differentiation. Cell. 1997;89(5):747-54.

6. Bi W, Deng JM, Zhang Z, Behringer RR, de Crombrugghe B. Sox9 is required for cartilage formation. Nat Genet. 1999;22(1):85-9.

7. Tontonoz P, Hu E, Graves RA, Budavari AI, Spiegelman BM. mPPAR gamma 2: tissue-specific regulator of an adipocyte enhancer. Genes Dev. 1994;8(10):1224-34.

8. Holliday R. Epigenetics: an overview. Dev Genet. 1994;15(6):453-7. 
9. Luger K, Mader AW, Richmond RK, Sargent DF, Richmond TJ. Crystal structure of the nucleosome core particle at 2.8 A resolution. Nature. 1997;389(6648):251-60.

10. Collinson A, Collier AJ, Morgan NP, Sienerth AR, Chandra T, Andrews S, et al. Deletion of the polycomb-group protein EZH2 leads to compromised self-renewal and differentiation defects in human embryonic stem cells. Cell Rep. 2016;17(10):2700-14.

11. Chamberlain SJ, Yee D, Magnuson T. Polycomb repressive complex 2 is dispensable for maintenance of embryonic stem cell pluripotency. Stem Cells. 2008;26(6):1496-505.

12. Pasini D, Bracken AP, Hansen JB, Capillo M, Helin K. The polycomb group protein Suz12 is required for embryonic stem cell differentiation. Mol Cell Biol. 2007;27(10):3769-79.

13.• Hemming S, Cakouros D, Isenmann S, Cooper L, Menicanin D, Zannettino A, et al. EZH2 and KDM6A act as an epigenetic switch to regulate mesenchymal stem cell lineage specification. Stem Cells. 2014;32(3):802-15 Hemming et al.'s study describes a unique epigenetic switch mediating the cell fate determination of BMSC between osteognesis and adipogenesis.

14. Ye L, Fan Z, Yu B, Chang J, Al Hezaimi K, Zhou X, et al. Histone demethylases KDM4B and KDM6B promotes osteogenic differentiation of human MSCs. Cell Stem Cell. 2012;11(1):50-61.

15. Yang D, Yu B, Sun H, Qiu L. The roles of histone demethylase Jmjd 3 in osteoblast differentiation and apoptosis. J Clin Med. 2017;6(3).

16. Yang D, Okamura H, Nakashima Y, Haneji T. Histone demethylase Jmjd3 regulates osteoblast differentiation via transcription factors Runx2 and osterix. J Biol Chem. 2013;288(47):33530-41.

17. Zhang F, Xu L, Xu L, Xu Q, Li D, Yang Y, et al. JMJD3 promotes chondrocyte proliferation and hypertrophy during endochondral bone formation in mice. J Mol Cell Biol. 2015;7(1):23-34.

18. Hemming S, Cakouros D, Codrington J, Vandyke K, Arthur A, Zannettino A, et al. EZH2 deletion in early mesenchyme compromises postnatal bone microarchitecture and structural integrity and accelerates remodeling. FASEB J. 2017;31(3):1011-27.

19. Dudakovic A, Camilleri ET, Paradise CR, Samsonraj RM, Gluscevic M, Paggi CA, et al. Enhancer of zeste homolog 2 (Ezh2) controls bone formation and cell cycle progression during osteogenesis in mice. J Biol Chem. 2018;293(33):12894-907.

20. Wang L, Jin Q, Lee JE, Su IH, Ge K. Histone H3K27 methyltransferase Ezh2 represses Wnt genes to facilitate adipogenesis. Proc Natl Acad Sci U S A. 2010;107(16):7317-22.

21. Jing H, Liao L, An Y, Su X, Liu S, Shuai Y, et al. Suppression of EZH2 prevents the shift of osteoporotic MSC fate to adipocyte and enhances bone formation during osteoporosis. Mol Ther. 2016;24(2):217-29.

22. Grandy RA, Whitfield TW, Wu H, Fitzgerald MP, VanOudenhove JJ, Zaidi SK, et al. Genome-wide studies reveal that H3K4me3 modification in bivalent genes is dynamically regulated during the pluripotent cell cycle and stabilized upon differentiation. Mol Cell Biol. 2016;36(4):615-27.

23. Voigt P, Tee WW, Reinberg D. A double take on bivalent promoters. Genes Dev. 2013;27(12):1318-38.

24. Yin B, Yu F, Wang C, Li B, Liu M, Ye L. Epigenetic control of mesenchymal stem cell fate decision via histone methyltransferase Ash1l. Stem Cells. 2019;37(1):115-27.

25. Tanaka Y, Kawahashi K, Katagiri Z, Nakayama Y, Mahajan M, Kioussis D. Dual function of histone H3 lysine 36 methyltransferase ASH1 in regulation of Hox gene expression. PLoS One. 2011;6(11):e28171.

26. Gregory GD, Vakoc CR, Rozovskaia T, Zheng X, Patel S, Nakamura T, et al. Mammalian ASH1L is a histone methyltransferase that occupies the transcribed region of active genes. Mol Cell Biol. 2007;27(24):8466-79.

27. Nayak A, Viale-Bouroncle S, Morsczeck C, Muller S. The SUMOspecific isopeptidase SENP3 regulates MLL1/MLL2 methyltransferase complexes and controls osteogenic differentiation. Mol Cell. 2014;55(1):47-58.

28. Ge W, Shi L, Zhou Y, Liu Y, Ma GE, Jiang Y, et al. Inhibition of osteogenic differentiation of human adipose-derived stromal cells by retinoblastoma binding protein 2 repression of RUNX2activated transcription. Stem Cells. 2011;29(7):1112-25.

29. Sepulveda H, Villagra A, Montecino M. Tet-Mediated DNA demethylation is required for SWI/SNF-dependent chromatin remodeling and histone-modifying activities that trigger expression of the Sp7 osteoblast master gene during mesenchymal lineage commitment. Mol Cell Biol. 2017;37(20).

30. Rojas A, Aguilar R, Henriquez B, Lian JB, Stein JL, Stein GS, et al. Epigenetic control of the bone-master Runx2 gene during osteoblast-lineage commitment by the histone demethylase JARID1B/KDM5B. J Biol Chem. 2015;290(47):28329-42.

31. Wang C, Wang J, Li J, Hu G, Shan S, Li Q, et al. KDM5A controls bone morphogenic protein 2-induced osteogenic differentiation of bone mesenchymal stem cells during osteoporosis. Cell Death Dis. 2016;7(8):e2335.

32. Kim HJ, Park JW, Lee KH, Yoon H, Shin DH, Ju UI, et al. Plant homeodomain finger protein 2 promotes bone formation by demethylating and activating Runx2 for osteoblast differentiation. Cell Res. 2014;24(10):1231-49.

33. Qi Q, Wang Y, Wang X, Yang J, Xie Y, Zhou J, et al. Histone demethylase KDM4A regulates adipogenic and osteogenic differentiation via epigenetic regulation of $\mathrm{C} / \mathrm{EBP} \alpha$ and canonical Wnt signaling. Cell Mol Life Sci. 2020;77(12):2407-21.

34. Lawson KA, Teteak CJ, Gao J, Li N, Hacquebord J, Ghatan A, et al. ESET histone methyltransferase regulates osteoblastic differentiation of mesenchymal stem cells during postnatal bone development. FEBS Lett. 2013;587(24):3961-7.

35. Matsumura $Y$, Nakaki R, Inagaki T, Yoshida A, Kano Y, Kimura $H$ et al. H3K4/H3K9me3 bivalent chromatin domains targeted by lineage-specific DNA methylation pauses adipocyte differentiation. Mol Cell. 2015;60(4):584-96.

36. Sinha KM, Yasuda H, Zhou X, de-Crombrugghe B. Osterix and NO66 histone demethylase control the chromatin of Osterix target genes during osteoblast differentiation. J Bone Miner Res. 2014;29(4):855-65.

37. Chen Q, Sinha K, Deng JM, Yasuda H, Krahe R, Behringer RR, et al. Mesenchymal deletion of histone demethylase NO66 in mice promotes bone formation. J Bone Miner Res. 2015;30(9):1608-17.

38. Yang X, Wang G, Wang Y, Zhou J, Yuan H, Li X, et al. Histone demethylase $\mathrm{KDM} 7 \mathrm{~A}$ reciprocally regulates adipogenic and osteogenic differentiation via regulation of $\mathrm{C} / \mathrm{EBP} \alpha$ and canonical Wnt signalling. J Cell Mol Med. 2019;23(3):2149-62.

39. Shahbazian MD, Grunstein M. Functions of site-specific histone acetylation and deacetylation. Annu Rev Biochem. 2007;76:75-100.

40. Bannister AJ, Kouzarides T. Regulation of chromatin by histone modifications. Cell Res. 2011;21(3):381-95.

41. Bradley EW, Carpio LR, van Wijnen AJ, McGee-Lawrence ME, Westendorf JJ. Histone deacetylases in bone development and skeletal disorders. Physiol Rev. 2015;95(4):1359-81.

42. Gordon JAR, Stein JL, Westendorf JJ, van Wijnen AJ. Chromatin modifiers and histone modifications in bone formation, regeneration, and therapeutic intervention for bone-related disease. Bone. 2015;81:739-45.

43. Carpio LR, Bradley EW, McGee-Lawrence ME, Weivoda MM, Poston DD, Dudakovic A, et al. Histone deacetylase 3 supports endochondral bone formation by controlling cytokine signaling and matrix remodeling. Sci Signal. 2016;9(440):ra79.

44.• Ali D, Hamam R, Alfayez M, Kassem M, Aldahmash A, Alajez NM. Epigenetic library screen identifies abexinostat as novel regulator of adipocytic and osteoblastic differentiation of human skeletal (mesenchymal) stem cells. Stem Cells Transl Med. 2016;5(8): 1036-47 Ali et al.'s study demonstrates the utility of high- 
throughput screening of chemical/peptide libraries to identify novel agents that may have therapeutic potential for modifying bone formation.

45.• Wang J, Wang CD, Zhang N, Tong WX, Zhang YF, Shan SZ, et al. Mechanical stimulation orchestrates the osteogenic differentiation of human bone marrow stromal cells by regulating HDAC1. Cell Death Dis. 2016;7:e2221 Wang et al.'s study highlights the role of external environmental simuli affecting the commitment of BMSC via changes in histone acetylation status.

46. Denu RA. SIRT3 enhances mesenchymal stem cell longevity and differentiation. Oxidative Med Cell Longev. 2017;2017:5841716.

47. Fukuda M, Yoshizawa T, Karim MF, Sobuz SU, Korogi W, Kobayasi D, et al. SIRT7 has a critical role in bone formation by regulating lysine acylation of SP7/Osterix. Nat Commun. 2018;9(1):2833.

48. Chen Z, Zhang Z, Guo L, Wei X, Zhang Y, Wang X, et al. The role of histone deacetylase 4 during chondrocyte hypertrophy and endochondral bone development. Bone Joint Res. 2020;9(2):82-9.

49. Rojas A, Sepulveda H, Henriquez B, Aguilar R, Opazo T, Nardocci G, et al. Mll-COMPASS complexes mediate H3K4me3 enrichment and transcription of the osteoblast master gene Runx $2 / p 57$ in osteoblasts. J Cell Physiol. 2019;234(5):6244-53.

50.• Sun J, Ermann J, Niu N, Yan G, Yang Y, Shi Y, et al. Histone demethylase LSD1 regulates bone mass by controlling WNT7B and BMP2 signaling in osteoblasts. Bone Res. 2018;6:14 Sun et al.'s study demonstrates the epigentic regulation of osteoblast function to increase bone mass via histone methylation.

51. Wang L, Niu N, Li L, Shao R, Ouyang H, Zou W. H3K36 trimethylation mediated by SETD2 regulates the fate of bone marrow mesenchymal stem cells. PLoS Biol. 2018;16(11):e2006522.

52.• Cakouros D, Hemming S, Gronthos K, Liu R, Zannettino A, Shi S, et al. Specific functions of TET1 and TET2 in regulating mesenchymal cell lineage determination. Epigenetics Chromatin. 2019;12(1):3 Cakouros et al.'s study highlights the importance of DNA methylation and hydroxymethylation in human BMSC proliferation and cell fate determination and the regulation of Tet molecules during osteoporosis.

53. Zhou GS, Zhang XL, Wu JP, Zhang RP, Xiang LX, Dai LC, et al. 5Azacytidine facilitates osteogenic gene expression and differentiation of mesenchymal stem cells by alteration in DNA methylation. Cytotechnology. 2009;60(1-3):11-22.

54. Yan X, Ehnert S, Culmes M, Bachmann A, Seeliger C, Schyschka $\mathrm{L}$, et al. 5-Azacytidine improves the osteogenic differentiation potential of aged human adipose-derived mesenchymal stem cells by DNA demethylation. PLoS One. 2014;9(6):e90846.

55. Eimori K, Endo N, Uchiyama S, Takahashi Y, Kawashima H, Watanabe K. Disrupted bone metabolism in long-term bedridden patients. PLoS One. 2016;11(6):e0156991.

56. Cappellesso R, Nicole L, Guido A, Pizzol D. Spaceflight osteoporosis: current state and future perspective. Endocr Regul. 2015;49(4):231-9.

57. Li Y, Ge C, Long JP, Begun DL, Rodriguez JA, Goldstein SA, et al. Biomechanical stimulation of osteoblast gene expression requires phosphorylation of the RUNX2 transcription factor. J Bone Miner Res. 2012;27(6):1263-74.

58. Zeng Z, Yin X, Zhang X, Jing D, Feng X. Cyclic stretch enhances bone morphogenetic protein-2-induced osteoblastic differentiation through the inhibition of Hey1. Int J Mol Med. 2015;36(5):1273-81.

59. Wang C, Shan S, Wang C, Wang J, Li J, Hu G, et al. Mechanical stimulation promote the osteogenic differentiation of bone marrow stromal cells through epigenetic regulation of Sonic Hedgehog. Exp Cell Res. 2017;352(2):346-56.

60. Wu X, Zhang Y. TET-mediated active DNA demethylation: mechanism, function and beyond. Nat Rev Genet. 2017;18(9):517-34.

61. Kriaucionis S, Heintz N. The nuclear DNA base 5hydroxymethylcytosine is present in Purkinje neurons and the brain. Science. 2009;324(5929):929-30.
62. Tahiliani M, Koh KP, Shen Y, Pastor WA, Bandukwala H, Brudno Y, et al. Conversion of 5-methylcytosine to 5-hydroxymethylcytosine in mammalian DNA by MLL partner TET1. Science. 2009;324(5929): 930-5.

63.• Yang R, Yu T, Kou X, Gao X, Chen C, Liu D, et al. Tet1 and Tet2 maintain mesenchymal stem cell homeostasis via demethylation of the P2rX7 promoter. Nat Commun. 2018;9(1):2143 Yang et al.'s study highlights the importance of Tet1 and 2 molecules in BMSC growth and differentiation, and their association with the development of osteoporosis.

64. Ito R, Shimada H, Yazawa K, Sato I, Imai Y, Sugawara A, et al. Hydroxylation of methylated DNA by TET1 in chondrocyte differentiation of C3H10T1/2 cells. Biochem Biophys Rep. 2016;5:134 40.

65. Taylor SE, Li YH, Smeriglio P, Rath M, Wong WH, Bhutani N. Stable 5-hydroxymethylcytosine $(5 \mathrm{hmC})$ acquisition marks gene activation during chondrogenic differentiation. J Bone Miner Res. 2016;31(3):524-34.

66. Nakashima K, Zhou X, Kunkel G, Zhang Z, Deng JM, Behringer $\mathrm{RR}$, et al. The novel zinc finger-containing transcription factor osterix is required for osteoblast differentiation and bone formation. Cell. 2002;108(1):17-29.

67. Zhang YX, Sun HL, Liang H, Li K, Fan QM, Zhao QH. Dynamic and distinct histone modifications of osteogenic genes during osteogenic differentiation. J Biochem. 2015;158(6):445-57.

68. Herlofsen SR, Bryne JC, Hoiby T, Wang L, Issner R, Zhang X, et al. Genome-wide map of quantified epigenetic changes during in vitro chondrogenic differentiation of primary human mesenchymal stem cells. BMC Genomics. 2013;14:105.

69. Cheung K, Barter MJ, Falk J, Proctor CJ, Reynard LN, Young DA. Histone ChIP-Seq identifies differential enhancer usage during chondrogenesis as critical for defining cell-type specificity. FASEB J. 2020;34(4):5317-31.

70. Mikkelsen TS, Xu Z, Zhang X, Wang L, Gimble JM, Lander ES, et al. Comparative epigenomic analysis of murine and human adipogenesis. Cell. 2010;143(1):156-69.

71. Meyer MB, Benkusky NA, Sen B, Rubin J, Pike JW. Epigenetic plasticity drives adipogenic and osteogenic differentiation of marrow-derived mesenchymal stem cells. J Biol Chem. 2016;291(34):17829-47.

72. Wu H, Gordon JA, Whitfield TW, Tai PW, van Wijnen AJ, Stein JL, et al. Chromatin dynamics regulate mesenchymal stem cell lineage specification and differentiation to osteogenesis. Biochim Biophys Acta Gene Regul Mech. 2017;1860(4):438-49.

73. Cao Y, Yang H, Jin L, Du J, Fan Z. Genome-wide DNA methylation analysis during osteogenic differentiation of human bone marrow mesenchymal stem cells. Stem Cells Int. 2018;2018:8238496.

74.• Dudakovic A, Camilleri ET, Riester SM, Paradise CR, Gluscevic M, O'Toole TM, et al. Enhancer of zeste homolog 2 inhibition stimulates bone formation and mitigates bone loss caused by ovariectomy in skeletally mature mice. J Biol Chem. 2016;291(47):24594-606 Dudakovic et al.'s study demonstrates the role of histone methylation in ospetoporosis and the therapeutic potential of epigenetic inhibitors to mitigate bone loss.

75. Zhang Y, Ma C, Liu X, Wu Z, Yan P, Ma N, et al. Epigenetic landscape in PPAR $\gamma 2$ in the enhancement of adipogenesis of mouse osteoporotic bone marrow stromal cell. Biochim Biophys Acta. 2015;1852(11):2504-16.

76. Li B, Zhao J, Ma JX, Li GM, Zhang Y, Xing GS, et al. Overexpression of DNMT1 leads to hypermethylation of H19 promoter and inhibition of Erk signaling pathway in disuse osteoporosis. Bone. 2018;111:82-91.

Publisher's Note Springer Nature remains neutral with regard to jurisdictional claims in published maps and institutional affiliations. 